Pacific

Journal of

Mathematics

\title{
CONSTANT-SPEED RAMPS
}

OSCAR M. PeRdomo

Volume 275 No. 1

May 2015 


\title{
CONSTANT-SPEED RAMPS
}

\author{
OSCAR M. PERdomo
}

\begin{abstract}
It is easy to show that if the kinetic coefficient of friction between a block and a ramp is $\mu_{k}$ and this ramp is a straight line with slope $-\mu_{k}$, then this block will move along the ramp with constant speed. A natural question to ask is the following: besides straight lines, are there other shapes of ramps such that a block will go down the ramp with constant speed? Here we classify all possible shapes of these ramps, and, surprisingly, we show that the planar ramps can be parametrized in terms of elementary functions: trigonometric functions, exponential functions and their inverses. They provide basic examples of curves explicitly parametrized by arclength. A video explaining the main results in this paper can be found at http://youtu.be/iBrvbb0efVk.
\end{abstract}

\section{Introduction}

It is known experimentally that when a block slides on a surface under the presence of gravity, besides the force exerted on the block by the surface - called the normal force - there is a second force on the block, with direction opposite to the direction of the velocity vector of the block and with length proportional to the length of the normal force. This second force is called the friction force. If we use symbols in a bold font to denote vectors and we use the same symbols (not in bold) to represent their magnitudes, then the friction force $\boldsymbol{F}$ satisfies

$$
F=\mu N,
$$

where $N$ is the normal force and $\mu$ is a constant called the kinetic coefficient of friction.

In this paper we will assume that (1-1) holds true, even though in real life it is just a good approximation and its formal deduction from basic physical laws is not known. Let us quote [Feynman et al. 1963] in this regard: "It is quite difficult to do accurate quantitative experiments in friction, and the laws of friction are still not analyzed very well, in spite of the enormous engineering value of an accurate analysis. Although the law $F=\mu N$ is fairly accurate once the surfaces are standardized, the reason for this form of the law is not really understood."

MSC2010: 53Z05.

Keywords: friction, constant speed, ramps, sliding. 


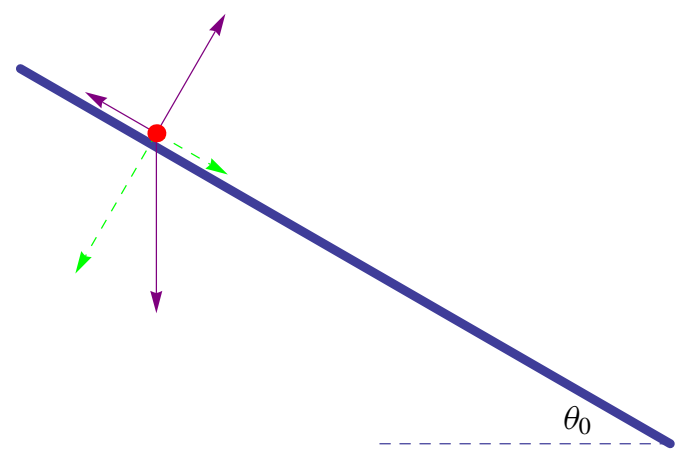

Figure 1. A block sliding down under the effect of gravity and the friction force. This figure shows the three forces acting on the block. The dashed forces are just a decomposition of the gravity force.

We will take (1-1) and the discussion of the direction of the forces in the previous paragraphs as a definition of the friction force and the kinetic coefficient of friction. One of the difficulties dealing with friction forces is that they are not continuous, due to the fact that their direction must be opposite to the velocity vector of the block. For example, friction forces are responsible for making a car stop; they produce a force opposite to the motion of the car, but once the car has stopped, this force disappears.

A basic computation shows that given a kinetic coefficient of friction $\mu_{k}$, the angle of a linear ramp can be adjusted so that a block on this ramp will move down with constant speed. In order to cancel the gravity, friction, and normal forces, it is enough to take a ramp given by a line with slope $-\mu_{k}$, that is, we must take the inclination of the ramp to be $\theta_{0}$ with $\tan \theta_{0}=\mu_{k}$. See Figure 1.

In this paper we study other ramps on which a block can slide down with constant speed under only the effect of gravity and the friction force. Let us mention some of the differences and similarities between the motion on a linear ramp and on these other ramps: (i) As happens for linear ramps, if a block is moving with constant speed on any ramp only under the effect of gravity and the friction force, then the velocity vector of the block must have a negative vertical component, that is, the motion must be downward (see Proposition 3.9). (ii) When the ramp is linear, the angle of the ramp (i.e., the shape of the ramp) is independent of the velocity of the block; it depends only on the coefficient of friction. For other ramps, the shape of the ramp depends on the desired constant speed of the block. Corollary 4.2 provides an exact procedure for changing the shape of the ramp according to the desired constant speed of the block.

Let us assume that we want a block to move down with speed $v_{0}$ and we know that the kinetic coefficient of friction is $\mu_{k}$. In this note, we classify the shapes of 


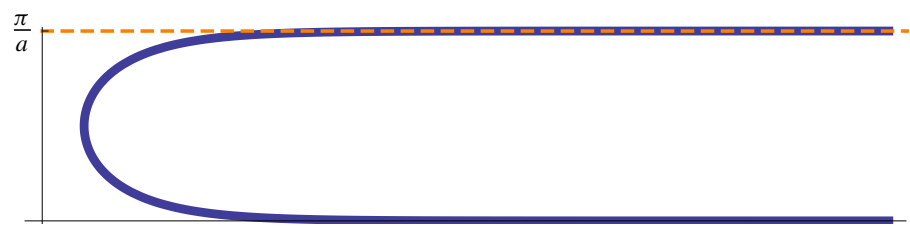

Figure 2. The graph of the curve $\boldsymbol{\alpha}$, which has two horizontal asymptotes, separated by a distance of $\pi / a$.

all possible ramps on which this block will move down with speed $v_{0}$. We now construct the 2-dimensional constant-speed ramp. Given $v_{0}$ and $\mu_{k}=\tan \delta$, define $a=g /\left(v_{0}^{2} \sin \delta\right)$, where $g$ is the acceleration due to gravity, and

$$
\boldsymbol{\alpha}(s)=\left(s+\frac{1}{a} \ln \left(1+\mathrm{e}^{-2 a s}\right), \frac{2}{a} \operatorname{arccot}\left(\mathrm{e}^{-a s}\right)\right) .
$$

This curve $\boldsymbol{\alpha}$ has a " $U$ " shape with two horizontal asymptotes: one at $y=0$ and the second one at $y=\pi / a$. See Figure 2 .

If we rotate the curve $\alpha$ clockwise through an angle $\delta$ (see Figure 3), then the highest point divides this rotated curve into two ramps, on which a block can move down with constant speed $v_{0}$ under the assumption that the kinetic coefficient of friction is $\mu_{k}=\tan \delta$.

The sequence of pictures in the Appendix shows the motion on the ramps when the desired speed is $5 \mathrm{~m} / \mathrm{s}$ and $\mu_{k}=0.5$. The pictures also display the three forces acting on the block under the assumption that the mass is $1 \mathrm{~kg}$ and $g=9.81 \mathrm{~m} / \mathrm{s}^{2}$ (recall that $g$ changes from place to place on earth). On the highest part of the ramp on the left, the normal force is zero. Also, although the normal force in the upper part of this ramp is pointing down, the block does not fall down due to its speed.

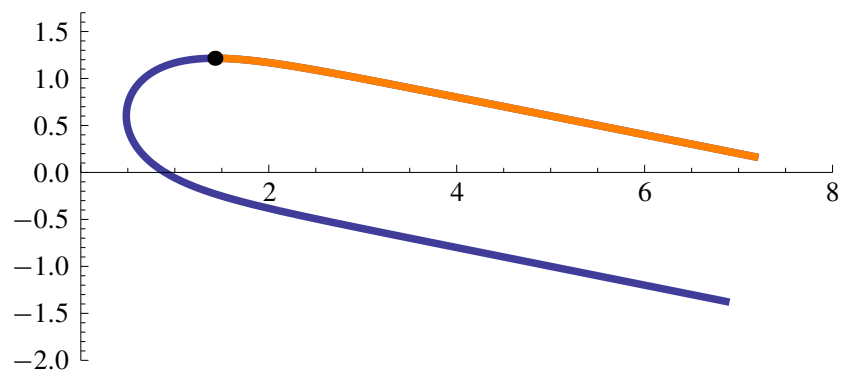

Figure 3. Graph of the two constant-speed ramps. At the highest point, a block with speed $v_{0}$ must be placed on top if we want to use the ramp to the right of this highest point, and it must be placed underneath if we want to use the ramp to the left of this highest point. 


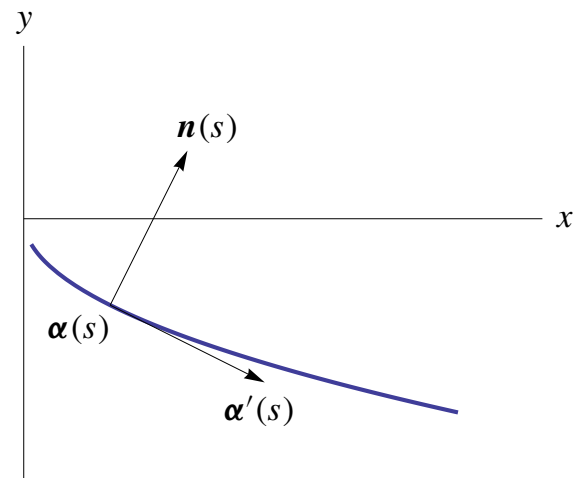

Figure 4. Given that $s$ is the arc-length parameter, the vectors $\boldsymbol{\alpha}^{\prime}(s)=(\cos \theta(s), \sin \theta(s))$ and $\boldsymbol{n}(s)=(-\sin \theta(s), \cos \theta(s))$ are perpendicular.

In Section 2 we provide a first proof of the classification of 2-dimensional ramps. In Section 3 we provide a formal definition of ramp in order to state the result as a mathematical theorem. This section may be skipped. Section 4 deals with 3-dimensional ramps.

\section{A first approach}

Let us find every possible 2-dimensional curve with the property that a block, sliding down on it, will move with constant speed $v>0$ under the assumption that the kinetic coefficient of friction is $\mu=\tan \delta$ for some constant $\delta$ between 0 and $\pi / 4$ radians. Start by assuming that this curve is parametrized by arclength; that is, if $\boldsymbol{\alpha}(s)=(x(s), y(s))$ denotes such a curve, then $\left|\boldsymbol{\alpha}^{\prime}(s)\right|=1$. Under this assumption we can assume that for a smooth function $\theta(s)$ we have

$$
x^{\prime}(s)=\cos \theta(s) \quad \text { and } \quad y^{\prime}(s)=\sin \theta(s) .
$$

The function $\theta(s)$ will help us describe the curve $\boldsymbol{\alpha}$. It is clear that the vector $\boldsymbol{n}(s)=(-\sin \theta(s), \cos \theta(s))$ is a unit vector perpendicular to $\boldsymbol{\alpha}^{\prime}(s)$, and the chain rule gives that $\boldsymbol{\alpha}^{\prime \prime}(s)=\theta^{\prime}(s) \boldsymbol{n}(s)$. Figure 4 shows these two vectors.

Let us assume that $\boldsymbol{\beta}(t)=\boldsymbol{\alpha}(v t)$ describes the motion of the block. Since $s=v t$ and $s$ is the arc-length parameter, the speed of the block is the constant $v$. Since we have that $\boldsymbol{\beta}^{\prime \prime}(t)=v^{2} \boldsymbol{\alpha}^{\prime \prime}(v t)$, we can write the free-body diagram for the constant-speed problem on $\boldsymbol{\alpha}$ with speed $v$ as in Figure 5.

As the free-body diagram shows, the following equation must hold true (a freebody diagram is essentially a picture that shows the forces acting on a body. For further discussion on free-body diagrams, see [Beer et al. 2010]):

$$
m v^{2} \theta^{\prime}(s) \boldsymbol{n}(s)=\lambda(s) \boldsymbol{n}(s)-\tan (\delta) \lambda(s) \boldsymbol{\alpha}^{\prime}(s)-(0, m g) .
$$




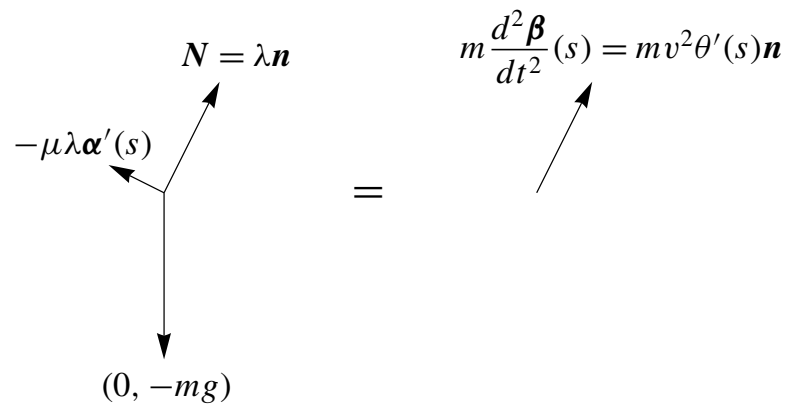

Figure 5. By Newton's second law, the sum of the normal force $\boldsymbol{N}$, the weight $(0,-m g)$ and the friction force $-\mu \lambda \boldsymbol{\alpha}^{\prime}(s)$ must be $m \boldsymbol{\beta}^{\prime \prime}(s)$.

By applying the inner product with the vector $\boldsymbol{\alpha}^{\prime}(s)$ to both sides of (2-1) we obtain

$$
\lambda(s)=-m g \cot \delta \sin \theta(s) .
$$

Likewise, applying the inner product with the vector $\boldsymbol{n}(s)$ to both sides of (2-1), we obtain that $m v^{2} \theta^{\prime}=\lambda-m g \cos \theta$. Therefore, with $a=g /\left(v^{2} \sin \delta\right)$,

$$
\theta^{\prime}(s)=\frac{-g}{v^{2} \sin \delta} \sin (\theta(s)+\delta)=-a \sin (\theta(s)+\delta) .
$$

Since the differential equation (2-2) does not have the variable $s$, all the solutions differ by a horizontal translation. That is, if $\theta(s)$ is a solution, then $\theta(s+c)$ is also a solution for every real number $c$. Due to the geometry of our problem we do not need to consider an integrating constant, since just one solution will give us all the solutions $\boldsymbol{\alpha}(s)$. Recall that the equilibrium solution of (2-2) is $\theta(s)=-\delta$ for all $s$. This solution corresponds to the case $\alpha(s)=(\cos (\delta) s,-\sin (\delta) s)$, which is the straight line ramp shown in Figure 1. When we solve this differential equation by separation of variables we notice that we need to integrate the function $\csc (\theta+\delta)$. Instead of using the classical formula $\int \csc u d u=-\ln (\csc u+\cot u)$ we will use the formula $\int \csc u d u=\ln (\tan (u / 2))$, which leads to the formula

$$
\theta(s)=-\delta+2 \arctan \left(\mathrm{e}^{-a s}\right) .
$$

It is clear that if $\boldsymbol{\gamma}(s)=(z(s), w(s))$ denotes a counterclockwise rotation of $\delta$ radians of the curve $\boldsymbol{\alpha}(s)$, then

$$
z^{\prime}(s)=\cos \left(2 \arctan \left(\mathrm{e}^{-a s}\right)\right) \quad \text { and } \quad w^{\prime}(s)=\sin \left(2 \arctan \left(\mathrm{e}^{-a s}\right)\right) .
$$

Integrating the equations above we obtain that

$$
z(s)=s+\frac{\ln \left(1+\mathrm{e}^{-2 a s}\right)}{a} \quad \text { and } \quad w(s)=\frac{2}{a} \operatorname{arccot}\left(\mathrm{e}^{-a s}\right) .
$$


The curve $(z(s), w(s))$ is shown in Figure 2. As mentioned in the introduction, the solution that we are looking for is a clockwise rotation of the curve $\gamma$ by an angle $\delta$. In the next section we give a more detailed explanation of this solution.

\section{Understanding the solution of the ODE: a mathematical definition of ramps}

With the intention of setting up notation, let us start with the following well-known definition (see, for example, [do Carmo 1976]).

Definition 3.1. We will say that a curve $\boldsymbol{\gamma}:\left[a_{1}, a_{2}\right] \rightarrow \mathbb{R}^{2}$ is regular if $\boldsymbol{\gamma}^{\prime}(t)$ never vanishes. We say $\boldsymbol{n}:\left[a_{1}, a_{2}\right] \rightarrow \mathbb{R}^{2}$ is a normal of the curve $\boldsymbol{\gamma}$ if $\boldsymbol{n}(t)$ has length 1 and the inner product of $\boldsymbol{n}(t)$ and $\boldsymbol{\gamma}^{\prime}(t)$ is zero, that is, $\boldsymbol{n}(t) \cdot \boldsymbol{\gamma}(t)=0$ for all $t$.

Intuitively, we can think of a planar ramp as a 2-dimensional region whose boundary is a curve. It is clear that the interesting part of the ramp is a portion of the boundary of the region. The following definition provides an alternative way to describe a ramp without mentioning the 2-dimensional region. Figure 6 provides the interpretation of our definition.

Definition 3.2. A ramp in the plane $\mathbb{R}^{2}$ is an ordered pair $(\boldsymbol{\gamma}, \boldsymbol{n})$, where $\boldsymbol{\gamma}$ : $\left[a_{1}, a_{2}\right] \rightarrow \mathbb{R}^{2}$ is a regular curve and $\boldsymbol{n}:\left[a_{1}, a_{2}\right] \rightarrow \mathbb{R}^{2}$ is a normal to $\boldsymbol{\gamma}$. We will interpret the ramp $(\boldsymbol{\gamma}, \boldsymbol{n})$ as a portion of the plane whose boundary contains $\boldsymbol{\gamma}$ and whose outer normal vector is $\boldsymbol{n}$.

Example 3.3. The pairs $\left(\boldsymbol{\gamma}_{1}, \boldsymbol{n}_{1}\right)$ and $\left(\boldsymbol{\gamma}_{2}, \boldsymbol{n}_{2}\right)$, mapping $[0, \pi] \rightarrow \mathbb{R}^{2}$ and given by

$$
\begin{array}{lll}
\boldsymbol{\gamma}_{1}(t)=5(\cos t, \sin t) & \text { and } & \boldsymbol{n}_{1}(t)=(\cos t, \sin t), \\
\boldsymbol{\gamma}_{2}(t)=5(\cos t, \sin t) & \text { and } & \boldsymbol{n}_{2}(t)=-(\cos t, \sin t),
\end{array}
$$

are examples of ramps, shown in Figure 6.

The following definition is based on Newton's second law:

Definition 3.4. A ramp with external force is a triple $(\boldsymbol{\gamma}, \boldsymbol{n}, \boldsymbol{F})$, where $\boldsymbol{F}:\left[a_{1}, a_{2}\right] \rightarrow$ $\mathbb{R}^{2}$ is a smooth function and $(\boldsymbol{\gamma}, \boldsymbol{n})$ is a ramp. Given a positive number $m$, a solution of the ramp with external force $(\boldsymbol{\gamma}, \boldsymbol{n}, \boldsymbol{F})$ is given by a curve $\boldsymbol{\beta}(t)=\boldsymbol{\gamma}(h(t))$ and a nonnegative function $\lambda:\left[a_{1}, a_{2}\right] \rightarrow \mathbb{R}$ such that

$$
\boldsymbol{F}(h(t))+\lambda(t) \boldsymbol{n}(h(t))=m \boldsymbol{\beta}^{\prime \prime}(t) .
$$

(Notice that $\boldsymbol{\beta}$ is just a reparametrization of the curve $\boldsymbol{\gamma}$ and is completely determined by the function $h:\left[a_{1}, a_{2}\right] \rightarrow \mathbb{R}$.)

Remark 3.5. In Definition 3.4, the term $\lambda(t) \boldsymbol{n}(t)$ represents the force that the surface of the ramp is exerting on the object under the action of the external force $\boldsymbol{F}$. By Newton's third law, $-\lambda(t) \boldsymbol{n}(t)$ is the force that the object is exerting on the 

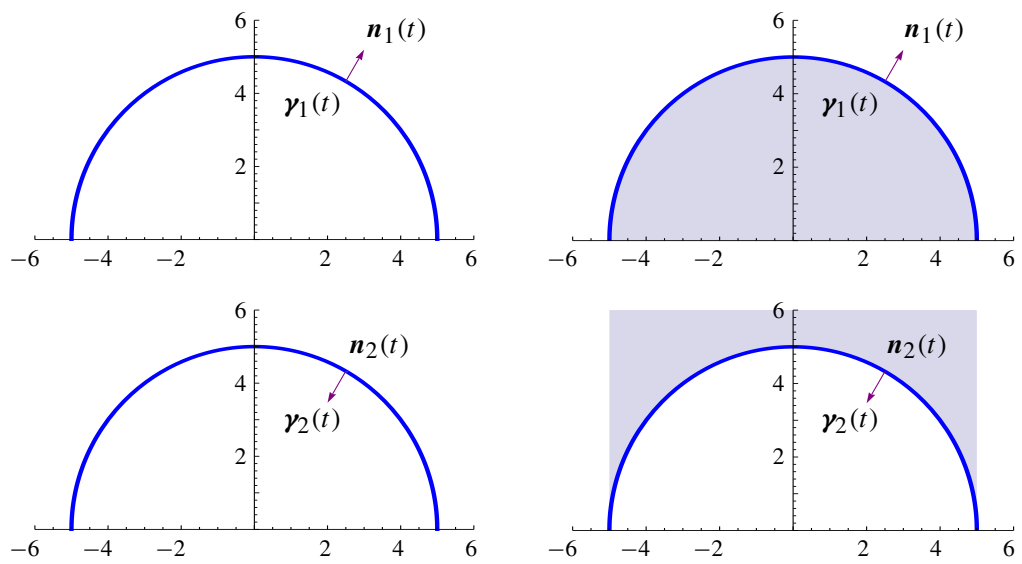

Figure 6. A normal vector of a curve helps us to define the part of a curve where we want a object to slide on a ramp

ramp. The condition $\lambda \geq 0$ is needed so that the object stays on the ramp. Also notice that the curve $\boldsymbol{\beta}(t)$ describes the position of the object at time $t$.

Example 3.6. If $(\boldsymbol{\gamma}, \boldsymbol{n})$ is a ramp, $m>0$ and $\boldsymbol{F}(t)=(0,-m g)$, where $g$ is the gravitational acceleration, then the triple $(\boldsymbol{\gamma}, \boldsymbol{n}, \boldsymbol{F})$ represents the action of gravity on a particle with mass $m$ that moves on the ramp without friction.

The following easy example illustrates that not every ramp with external force has a solution.

Example 3.7. Let us consider the ramp $(\boldsymbol{\gamma}, \boldsymbol{n})$ where $\boldsymbol{\gamma}, \boldsymbol{n}:[-1.5,1.5] \rightarrow \mathbb{R}^{2}$ are given by

$$
\boldsymbol{\gamma}(t)=\left(t, t^{2}\right) \quad \text { and } \quad \boldsymbol{n}(t)=\left(\frac{2 t}{\sqrt{4 t^{2}+1}}, \frac{-1}{\sqrt{4 t^{2}+1}}\right) .
$$

If we assume that the mass of the block is $1 \mathrm{~kg}$, and $\boldsymbol{F}(t)=(0,-9.81)$, then the ramp with external force $(\boldsymbol{\gamma}, \boldsymbol{n}, \boldsymbol{F})$ has no solution. Figure 7 shows this ramp.

Proof. Let us argue by contradiction. If a solution exists, there will exist a positive function $\lambda(t)$ and a function $h(t)$ such that

$$
\begin{aligned}
(0,-9.81)+\lambda(t)\left(\frac{2 h(t)}{\sqrt{4 h^{2}(t)+1}}, \frac{-1}{\sqrt{4 h^{2}(t)+1}}\right) & =\boldsymbol{\beta}^{\prime \prime}(t) \\
& =\left(h^{\prime \prime}(t), 2\left(h^{\prime}(t)\right)^{2}+2 h(t) h^{\prime \prime}(t)\right) .
\end{aligned}
$$

If we take the dot product with the vector $(2 h(t),-1)$ on both sides of the equation above we obtain the equation

$$
9.81+\lambda(t) \sqrt{4 h^{2}(t)+1}=-2\left(h^{\prime}(t)\right)^{2} .
$$

This is impossible because we assumed that $\lambda(t)$ is a positive function. 


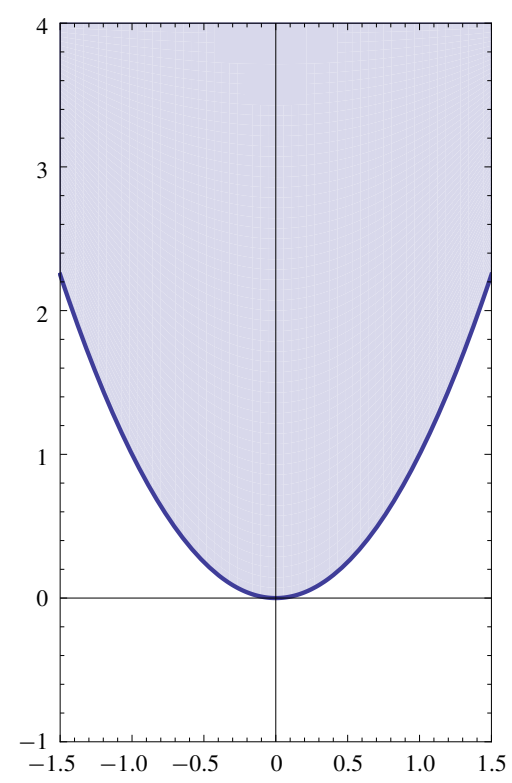

Figure 7. This ramp with the external force $\boldsymbol{F}(t)=(0,-9.81)$ does not have a solution.

The definition of the solution of a particle moving on the ramp under the action of the force $\boldsymbol{F}$ is provided by Definition 3.4. In the case that $\boldsymbol{F}$ denotes all the forces acting on the particle that moves on the ramp except the friction force, the definition of the solution needs to be changed to:

Definition 3.8. Given a ramp with external force $(\boldsymbol{\gamma}, \boldsymbol{n}, \boldsymbol{F})$ defined on the interval $\left[a_{1}, a_{2}\right]$ and two positive numbers $\mu<1$ and $m$, a solution of the ramp with external force $(\boldsymbol{\gamma}, \boldsymbol{n}, \boldsymbol{F})$ and kinetic coefficient of friction $\mu$ is given by a curve $\boldsymbol{\beta}(t)=\boldsymbol{\gamma}(h(t))$ and a nonnegative function $\lambda:\left[a_{1}, a_{2}\right] \rightarrow \mathbb{R}$ such that

$$
\boldsymbol{F}(h(t))+\lambda(t) \boldsymbol{n}(h(t))-\mu \lambda(t) \frac{\boldsymbol{\beta}^{\prime}(t)}{\left|\boldsymbol{\beta}^{\prime}(t)\right|}=m \boldsymbol{\beta}^{\prime \prime}(t) .
$$

Intuitively, when we think of a block moving with constant speed on a ramp, we think of the block moving down. The following proposition shows, using Definition 3.8, that indeed the block must move down.

Proposition 3.9. Let us assume that $\boldsymbol{F}=(0,-m g)$ is the gravitational force, where $m$ is a mass, and $\mu$ is a kinetic coefficient of friction. Let us further assume that $\boldsymbol{\beta}(t)=\boldsymbol{\gamma}(h(t))$ is a solution of the ramp with external force $(\boldsymbol{\gamma}, \boldsymbol{n}, \boldsymbol{F})$ and kinetic coefficient of friction $\mu$. If the speed of the solution is constant, then the velocity vector of the solution $\boldsymbol{\beta}$ cannot point up; that is, for any $t$, the dot product $\boldsymbol{\beta}^{\prime}(t) \cdot(0,1)$ cannot be positive. 
Proof. Since we are assuming that the curve $\boldsymbol{\gamma}$ is regular, we can find a reparametrization $\tilde{\gamma}$ that is parametrized by arclength (see for example [do Carmo 1976]). That is, we can write $\boldsymbol{\gamma}(t)=\tilde{\boldsymbol{\gamma}}(u(t))$. We therefore have that

$$
\boldsymbol{\beta}(t)=\boldsymbol{\gamma}(h(t))=\tilde{\boldsymbol{\gamma}}(u(h(t))=\tilde{\boldsymbol{\gamma}}(\tilde{h}(t)), \quad \text { where } \tilde{h}=u \circ h .
$$

Since $\tilde{\boldsymbol{\gamma}}$ is parametrized by arclength and we are assuming that the speed of $\boldsymbol{\beta}$ is constant, we have that the function $\tilde{h}^{\prime}$ is constant; that is $\tilde{h}^{\prime}(t)=v$ for all $t$ and for some nonzero real number $v$. Therefore $\boldsymbol{\beta}^{\prime \prime}(t)=v^{2} \tilde{\boldsymbol{\gamma}}^{\prime \prime}(t)$. Since we are assuming that $\boldsymbol{\beta}$ is a solution of the ramp with external force $(\boldsymbol{\gamma}, \boldsymbol{n}, \boldsymbol{F})$ and kinetic coefficient of friction $\mu$,

$$
(0,-m g)+\lambda(t) \boldsymbol{n}(h(t))-\mu \lambda(t) \frac{\boldsymbol{\beta}^{\prime}(t)}{\left|\boldsymbol{\beta}^{\prime}(t)\right|}=m \boldsymbol{\beta}^{\prime \prime}(t) .
$$

Multiplying the equation above by the velocity vector $\boldsymbol{\beta}^{\prime}(t)=v \tilde{\boldsymbol{\gamma}}^{\prime}(t)$ and keeping in mind that (i) this velocity vector is perpendicular to the normal vector $\boldsymbol{n}$ and (ii) the acceleration vector $\boldsymbol{\beta}^{\prime \prime}$ is parallel to the normal vector $\boldsymbol{n}$, we obtain

$$
-m g \boldsymbol{\beta}^{\prime}(t) \cdot(0,1)-\mu \lambda(t)|v|=0 .
$$

From the equation above we conclude that $\boldsymbol{\beta}^{\prime}(t) \cdot(0,1)$ cannot be positive.

Remark 3.10. Let us define an elementary function to be a function of one variable with real values built from a finite number of trigonometric, exponential, constant, $n$-th power functions and their inverses, through composition and the four basic elementary operations $(+,-, \times, \div)$. When we want to define basic examples of curves parametrized by arclength (that is, if we want to define $\boldsymbol{\gamma}(s)=(x(s), y(s))$ such that $x^{\prime}(s)^{2}+y^{\prime}(s)^{2}=1$ for all $\left.t\right)$, the first thing that comes to mind is to find easy possibilities for $x^{\prime}(t)$ and $y^{\prime}(t)$. The easiest one would be a pair of numbers $c_{1}$ and $c_{2}$ such that $c_{1}^{2}+c_{2}^{2}=1$. If we make $x^{\prime}(s)=c_{1}$ and $y^{\prime}(s)=c_{2}$, then, after an easy integration, we obtain that the curve $\boldsymbol{\gamma}$ is a straight line. If we want to use the fact that $\cos ^{2}(a s)+\sin ^{2}(a s)=1$ and we decide to make $x^{\prime}(s)=\cos (a s)$ and $y^{\prime}(s)=\sin (a s)$, then, after an easy integration, we obtain that $\boldsymbol{\gamma}$ should be a circle. The most important curves in this paper are those that are obtained by using the identity

$$
\tanh ^{2} t+\operatorname{sech}^{2} t=1 \quad \text { for all } t .
$$

That is, we will be using curves that satisfy $x^{\prime}(s)=\tanh ($ as $)$ and $y^{\prime}(s)=\operatorname{sech}($ as $)$.

The following lemma is a direct computation and provides a definition of the curve $\boldsymbol{\alpha}$ whose graph is shown in Figure 2 . 
Lemma 3.11. For any nonzero real number a, the curve

$$
\boldsymbol{\alpha}(s)=(x(s), y(s))=\left(s+\frac{1}{a} \ln \left(1+\mathrm{e}^{-2 a s}\right), \frac{2}{a} \operatorname{arccot}\left(\mathrm{e}^{-a s}\right)\right)
$$

is an arc-length parametrized curve. Moreover,

$$
x^{\prime}(s)=\tanh (\text { as }) \quad \text { and } \quad y^{\prime}(s)=\operatorname{sech}(\text { as }) .
$$

Lemma 3.12. For any positive number $\delta$ smaller than $\pi / 2$, suppose $\boldsymbol{\alpha}_{\delta}$ represents the curve obtained by rotating the curve $\boldsymbol{\alpha}=(x(s), y(s))$ defined in Lemma 3.11 through an angle of $\delta$ radians; that is, let

$$
\begin{aligned}
\boldsymbol{\alpha}_{\delta}(s) & =\left(x_{\delta}(s), y_{\delta}(s)\right) \\
& =(\cos (\delta) x(s)+\sin (\delta) y(s),-\sin (\delta) x(s)+\cos (\delta) y(s)) .
\end{aligned}
$$

Then the maximum value for $y_{\delta}$ is achieved when $s=\operatorname{arcsinh}(\cot \delta) / a$. Also, we have that

$$
\boldsymbol{\alpha}_{\delta}^{\prime \prime}(s) \cdot\left(y_{\delta}^{\prime}(s),-x_{\delta}^{\prime}(s)\right)=a \operatorname{sech}(a s) .
$$

The graph of the curve $\boldsymbol{\alpha}_{\delta}$ is shown in Figure 3.

Proof. A direct computation using Lemma 3.11 shows that

$$
y_{\delta}^{\prime}(s)=-\sin \delta \tanh (a s)+\cos \delta \operatorname{sech}(a s),
$$

and so the only solution of the equation $y_{\delta}^{\prime}(s)=0$ is $s=\operatorname{arcsinh}(\cot \delta) / a$. In order to prove the identity (3-2) we point out that since the inner product is invariant under rotations, this identity is equivalent to showing that $\boldsymbol{\alpha}^{\prime \prime}(s) \cdot\left(y^{\prime}(s),-x^{\prime}(s)\right)=$ $a \operatorname{sech}(a s)$, which follows because

$$
\begin{aligned}
x^{\prime \prime}(s) y^{\prime}(s)-y^{\prime \prime}(s) x^{\prime}(s) & =a \operatorname{sech}^{3} a s+a \tanh ^{2} \text { as sech } a s \\
& =a \operatorname{sech} a s\left(\operatorname{sech}^{2} a s+\tanh ^{2} a s\right)=a \operatorname{sech} a s .
\end{aligned}
$$

Remark 3.13. In this proof, we obtained $y_{\delta}^{\prime}(s)=-\sin \delta \operatorname{sech}(a s)(\sinh (a s)-\cot \delta)$. It follows that $y_{\delta}^{\prime}(s)>0$ if $s<s_{0}$ and $y_{\delta}^{\prime}(s)<0$ if $s>s_{0}$.

Definition 3.14. For any positive number $\delta$ smaller than $\pi / 2$ and any $a>0$, we define the ramps $\left(\boldsymbol{\gamma}_{\delta}, \boldsymbol{n}_{\delta}\right)$ and $\left(\tilde{\boldsymbol{\gamma}}_{\delta}, \tilde{\boldsymbol{n}}_{\delta}\right)$ on the interval $[0, \infty)$ by

$$
\begin{array}{lll}
\boldsymbol{\gamma}_{\delta}(s)=\boldsymbol{\alpha}_{\delta}\left(s_{0}-s\right), & \boldsymbol{n}_{\delta}(s)=\left(y_{\delta}^{\prime}\left(s_{0}-s\right),-x_{\delta}^{\prime}\left(s_{0}-s\right)\right), \\
\tilde{\boldsymbol{\gamma}}_{\delta}(s)=\boldsymbol{\alpha}_{\delta}\left(s+s_{0}\right), & \tilde{\boldsymbol{n}}_{\delta}(s)=\left(-y_{\delta}^{\prime}\left(s+s_{0}\right), x_{\delta}^{\prime}\left(s+s_{0}\right)\right),
\end{array}
$$

where $s_{0}=\operatorname{arcsinh}(\cot \delta) / a$ and the map $\boldsymbol{\alpha}_{\delta}$ and the functions $x_{\delta}$ and $y_{\delta}$ are defined in Lemma 3.12. These two ramps are shown in Figure 8.

Let us state and proof the main theorem in this section: 

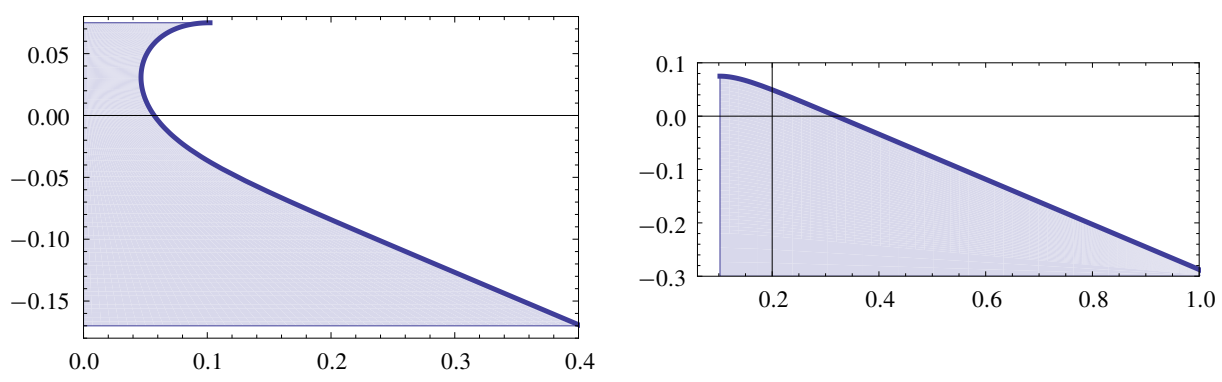

Figure 8. The two ramps given in Definition 3.14.

Theorem 3.15. Given an angle $\delta$ between 0 and $\pi / 4$ radians and $a$ speed $v>0$, set $\mu=\tan \delta, a=g /\left(v^{2} \sin \delta\right)$ and $\boldsymbol{F}(t)=(0,-m g)$, where $g$ is the acceleration due to gravity. We have:

(a) For the ramp $\left(\boldsymbol{\gamma}_{\delta}, \boldsymbol{n}_{\delta}\right)$ given in Definition 3.14,

$$
\boldsymbol{\beta}(t)=\boldsymbol{\gamma}_{\delta}(v t) \quad \text { and } \quad \lambda(t)=m g \cot \delta y_{\delta}^{\prime}\left(s_{0}-v t\right)
$$

is a solution for the ramp with external force $\left(\boldsymbol{\gamma}_{\delta}, \boldsymbol{n}_{\delta}, \boldsymbol{F}\right)$ and kinetic coefficient of friction $\tan \delta$.

(b) For the ramp $\left(\tilde{\boldsymbol{\gamma}}_{\delta}, \tilde{\boldsymbol{n}}_{\delta}\right)$ given in Definition 3.14,

$$
\boldsymbol{\beta}(t)=\tilde{\boldsymbol{\gamma}}_{\delta}(v t) \quad \text { and } \quad \lambda(t)=-m g \cot \delta y_{\delta}^{\prime}\left(s_{0}+v t\right)
$$

is a solution for the ramp with external force $\left(\tilde{\boldsymbol{\gamma}}_{\delta}, \tilde{\boldsymbol{n}}_{\delta}, \boldsymbol{F}\right)$ and kinetic coefficient of friction $\tan \delta$.

(c) Since in both cases $\left|\boldsymbol{\beta}^{\prime}(t)\right|=v$, these motions have constant speed.

Proof. Let us prove part (a). First of all, notice that, as is required by Definition 3.8, $\lambda>0$ by Remark 3.13. From the definition of $\boldsymbol{\beta}$ we obtain that

$$
\boldsymbol{\beta}^{\prime}(t)=-v \boldsymbol{\alpha}_{\delta}^{\prime}\left(s_{0}-v t\right) \quad \text { and } \quad \boldsymbol{\beta}^{\prime \prime}(t)=v^{2} \boldsymbol{\alpha}_{\delta}^{\prime \prime}\left(s_{0}-v t\right) .
$$

Therefore, the equation

$$
\boldsymbol{F}(h(t))+\lambda(t) \boldsymbol{n}(h(t))-\mu \lambda(t) \frac{\boldsymbol{\beta}^{\prime}(t)}{\left|\boldsymbol{\beta}^{\prime}(t)\right|}=m \boldsymbol{\beta}^{\prime \prime}(t)
$$

is equivalent to

$$
(0,-m g)+\lambda(t) \boldsymbol{n}_{\delta}(v t)+\tan \delta \lambda(t) \boldsymbol{\alpha}_{\delta}^{\prime}\left(s_{0}-v t\right)=m v^{2} \boldsymbol{\alpha}_{\delta}^{\prime \prime}(t) .
$$

Notice that the vectors $\boldsymbol{u}_{1}=\boldsymbol{n}_{\delta}(v t)$ and $\boldsymbol{u}_{2}=\boldsymbol{\alpha}_{\delta}^{\prime}\left(s_{0}-t v\right)$ form an orthonormal basis. Therefore, in order to establish (3-3) it is enough to prove that the dot products 
with $\boldsymbol{u}_{1}$ and $\boldsymbol{u}_{2}$ of the left-hand side and right-hand side of the equation are the same. The dot product of the LHS of (3-3) with $\boldsymbol{u}_{1}$ is equal to

$$
\begin{aligned}
m g x_{\delta}^{\prime}\left(s_{0}-v t\right)+\lambda(t)= & m g x_{\delta}^{\prime}\left(s_{0}-v t\right)+m g \cot \delta y_{\delta}^{\prime}\left(s_{0}-v t\right) \\
= & m g\left(\cos \delta \tanh \left(a\left(s_{0}-v t\right)\right)+\sin \delta \operatorname{sech}\left(a\left(s_{0}-v t\right)\right)\right) \\
& +m g \cot \delta\left(-\sin \delta \tanh \left(a\left(s_{0}-v t\right)\right)+\cos \delta \operatorname{sech}\left(a\left(s_{0}-v t\right)\right)\right) \\
= & \frac{m g}{\sin \delta} \operatorname{sech}\left(a\left(s_{0}-v t\right)\right) .
\end{aligned}
$$

Using (3-2) we get that the dot product of the RHS of (3-3) with $\boldsymbol{u}_{1}$ is equal to

$$
m v^{2} a \operatorname{sech}\left(a\left(s_{0}-v t\right)\right)=\frac{m g}{\sin \delta} \operatorname{sech}\left(a\left(s_{0}-v t\right)\right) .
$$

Therefore, LHS $\cdot \boldsymbol{u}_{1}=$ RHS $\cdot \boldsymbol{u}_{1}$. It is easy to check that the dot product of the RHS of (3-3) with $\boldsymbol{u}_{2}$ vanishes. On the other hand, the dot product of the LHS of (3-3) with $\boldsymbol{u}_{2}$ equals

$$
-m g y_{\delta}^{\prime}\left(s_{0}-v t\right)+v \tan \delta \lambda=-m g y_{\delta}^{\prime}\left(s_{0}-v t\right)+\tan \delta m g \cot \delta y_{\delta}^{\prime}\left(s_{0}-v t\right)=0 .
$$

Therefore, part (a) follows. Part (b) is similar. Part (c) follows because $\left|\boldsymbol{\alpha}^{\prime}\right|=1$, and, since $\boldsymbol{\alpha}_{\delta}$ is a rotation of $\boldsymbol{\alpha}$, we have $\left|\boldsymbol{\alpha}_{\delta}^{\prime}\right|=1$ and $\boldsymbol{\beta}^{\prime}(t)=-v \boldsymbol{\alpha}^{\prime}\left(s_{0}-v t\right)$, so $\left|\boldsymbol{\beta}^{\prime}(t)\right|=v$.

\section{Three-dimensional ramps}

In this section we describe ramps in the space on which an object can move with constant speed $v_{0}$ under the assumption that the kinetic coefficient of friction is $\mu$. We will prove that, if $v_{0}$ and $\mu$ are fixed, then for every continuously differentiable unit tangent vector field in the southern hemisphere there will correspond a constantspeed family of ramps. In the correspondence that we will establish, the ramp that we defined in Section 2 corresponds to a particular choice of a tangent vector field.

For curves that represent planar ramps, we obtained a description by studying their velocity vector. Recall that the function $\theta(s)$ was used to describe a point in the lower part of the semicircle that represented a possible velocity vector of the curve that described the ramp. When this curve is free to move in the whole 3-dimensional space, the tangent unit vector to the curve lies in the unit sphere, and since the block must go down the ramp we can assume that the tangent unit vector of the ramp lies in the southern hemisphere. See Figure 9.

In order to completely determine the ramp, we need to specify the desired direction of the normal to the ramp; see Figure 10. We can do this by choosing a unit normal vector field $\boldsymbol{H}(y)$ defined in the lower hemisphere and requesting the normal vector of the ramp to be equal to $N(y)$ any time the tangent unit vector to the ramp is the vector $y$. See Figure 11 . 

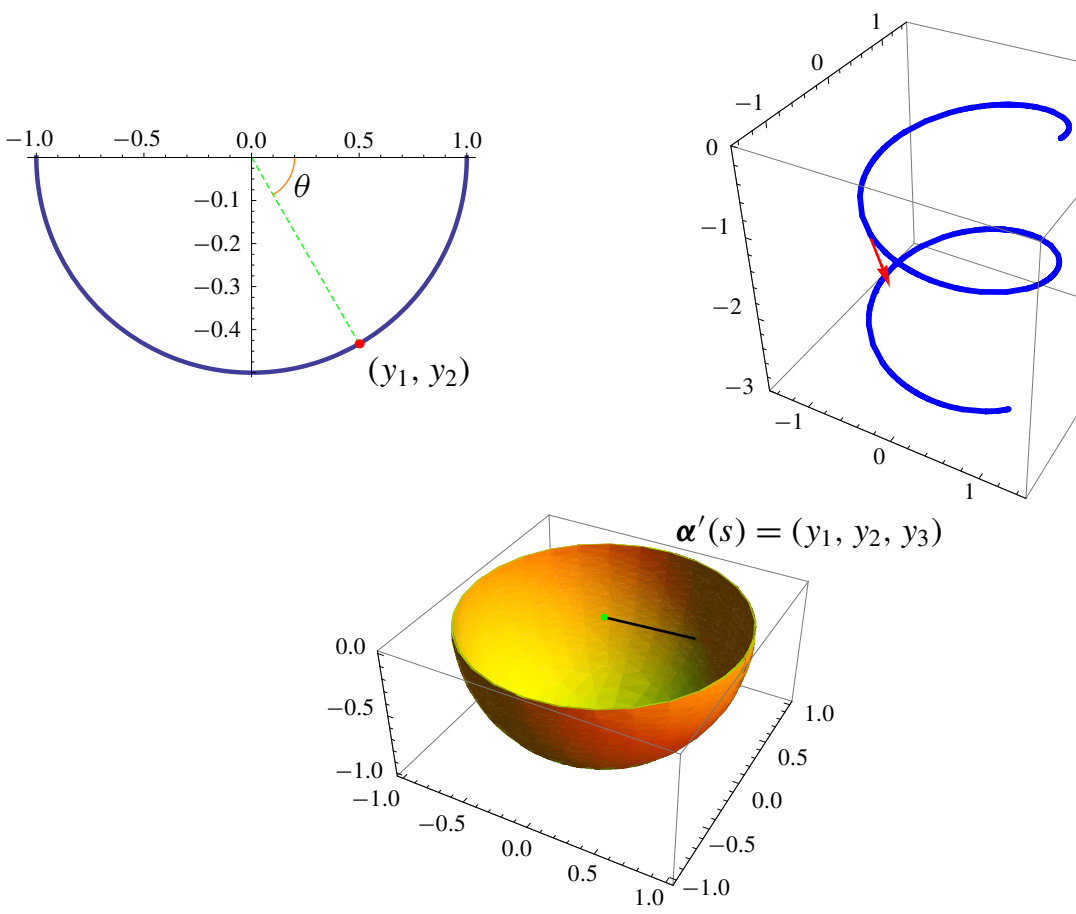

Figure 9. The unit tangent vector of a possible ramp in the plane lies in the lower part of the unit semicircle. If the ramp does not lie in a plane, but instead in the whole space, then the unit tangent vector lies in the lower hemisphere of the unit sphere.

We will prove that for any continuously differentiable choice of a normal field $\boldsymbol{n}(s)$ in the lower hemisphere, there exists a family of ramps on which a motion with constant speed is possible under the effect of the forces of gravity and friction.

More precisely, we have the following theorem:

Theorem 4.1. Let $\Sigma=\left\{\left(y_{1}, y_{2}, y_{3}\right) \in \mathbb{R}^{3}: y_{1}^{2}+y_{2}^{2}+y_{3}^{2}=1\right.$ and $\left.y_{3} \leq 0\right\}$ be the southern hemisphere and let $N: \Sigma \rightarrow \mathbb{R}^{3}$ be any continuously differentiable unit tangent vector field. That is, for any $y \in \Sigma, N(y)$ has norm 1 and is perpendicular to $y$. For any positive numbers $m, v$ and $\mu<1$, and any $y_{0} \in \Sigma$, there exists a unique curve $\boldsymbol{\alpha}(s)$ parametrized by arclength, such that $\boldsymbol{\alpha}(0)=(0,0,0)$ and $\boldsymbol{\alpha}^{\prime}(0)=y_{0}$. Furthermore, the motion $\boldsymbol{\beta}(t)=\boldsymbol{\alpha}(v t)$ in the ramp given by

$$
\boldsymbol{R}(s, r)=\boldsymbol{\alpha}(s)+r \boldsymbol{\alpha}^{\prime}(s) \times \boldsymbol{N}\left(\boldsymbol{\alpha}^{\prime}(s)\right)
$$

represents a solution of Newton's second law for a block with mass $m$ moving on the ramp under the assumption that the only forces acting on the block are gravity and friction with kinetic constant coefficient $\mu$. Recall that since the parameter $s$ is the arclength, $\left|\boldsymbol{\beta}^{\prime}(t)\right|=v$ for all $t$; that is, the motion has constant speed $v$. 

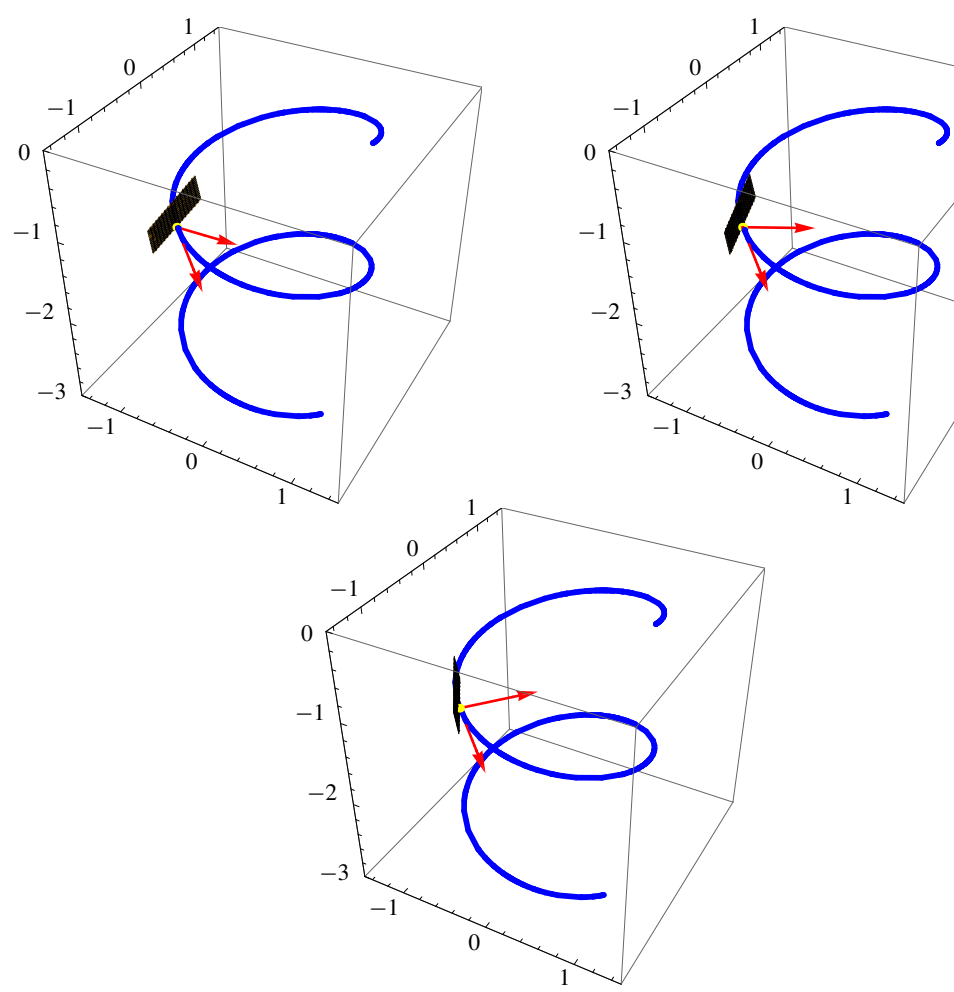

Figure 10. To build a ramp we need to choose a normal direction at every point in the ramp.

Proof. Recall that we denote by $\boldsymbol{u} \cdot \boldsymbol{w}$ the inner product of the vectors $\boldsymbol{u}$ and $\boldsymbol{w}$. A direct computation shows that if $\boldsymbol{e}_{3}=(0,0,1)$, then $\boldsymbol{e}_{3}^{T}: \Sigma \rightarrow \mathbb{R}^{3}$ given by $\boldsymbol{e}_{3}^{T}(y)=\boldsymbol{e}_{3}-\left(\boldsymbol{e}_{3} \cdot y\right) y$ is a tangent vector field. Notice that $\boldsymbol{e}_{3}^{T}(y)$ must be in the tangent space $T_{y} \Sigma$ because $\boldsymbol{e}_{3}^{T}(y) \cdot y=0$.

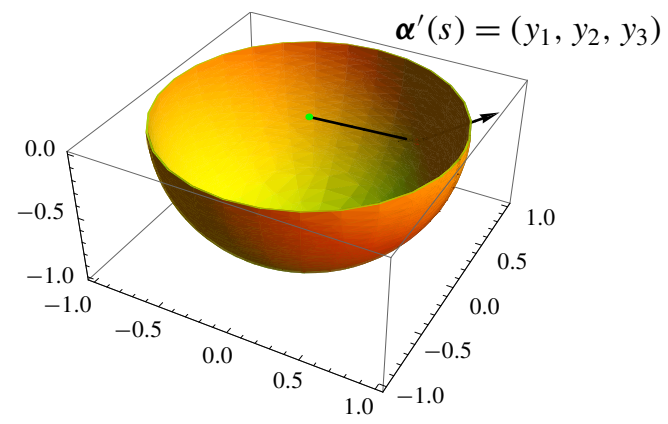

Figure 11. We will make the choice of the normal direction on the ramp to depend on the unit tangent vector. 
Let $\lambda: \Sigma \rightarrow \mathbb{R}$ be the function given by

$$
\lambda(y)=-\frac{g}{\mu} \boldsymbol{e}_{3} \cdot y=-\frac{g}{\mu} y_{3} .
$$

Since $y_{3} \leq 0, \lambda \geq 0$ and $\lambda$ only vanishes on the boundary of $\Sigma$. (Recall that the boundary of $\Sigma$ is given by the equation $y_{3}=0$.) Let us consider the tangent vector field

$$
\boldsymbol{X}=-\frac{1}{v^{2}}\left(g \boldsymbol{e}_{3}^{T}-\lambda \boldsymbol{N}(y)\right)=-\frac{g}{v^{2}}\left(\boldsymbol{e}_{3}^{T}+\frac{y_{3}}{\mu} \boldsymbol{N}(y)\right) .
$$

Observe that along points in the boundary of $\Sigma, \boldsymbol{X}(y)=-\left(g / v^{2}\right) \boldsymbol{e}_{3}$ points toward $\Sigma$. It follows that any integral curve of the tangent vector field $\boldsymbol{X}$ remains in $\Sigma$. Let $\boldsymbol{\gamma}(s)$ be the integral curve of the vector field $\boldsymbol{X}$ that satisfies $\boldsymbol{\gamma}(0)=y_{0}$. We will prove the theorem by showing the curve $\boldsymbol{\alpha}(s)$ given by

$$
\boldsymbol{\alpha}(s)=\int_{0}^{s} \boldsymbol{\gamma}(u) d u
$$

satisfies all the conditions in the theorem.

Clearly, $\boldsymbol{\alpha}(0)=(0,0,0)$ and $\boldsymbol{\alpha}^{\prime}(0)=\boldsymbol{\gamma}(0)=y_{0}$. We also have that $s$ is an arc-length parameter because $\left|\boldsymbol{\alpha}^{\prime}(s)\right|=|\boldsymbol{\gamma}(s)|=1$. A direct computation shows that the normal vector of the surface (or ramp) $\boldsymbol{R}$ along the curve $\boldsymbol{\alpha}$ (along points in the surface with $r=0$ ) is given by $N(\gamma(s))$.

We will now prove that Newton's second law holds true for $\boldsymbol{\beta}(t)=\boldsymbol{\alpha}(v t)$. Notice that since $v$ is constant, $\boldsymbol{\beta}^{\prime}(t)=v \boldsymbol{\alpha}^{\prime}(v t)=v \boldsymbol{\gamma}(s)$ and $\boldsymbol{\beta}^{\prime \prime}(v t)=v^{2} \boldsymbol{\gamma}^{\prime}(v t)=v^{2} \boldsymbol{\gamma}^{\prime}(s)$. If $m$ denotes the mass of the particle and $\lambda$ is defined as in the beginning of the proof, we have

$$
\begin{aligned}
m \boldsymbol{\beta}^{\prime \prime}(t)=m v^{2} \boldsymbol{\gamma}^{\prime}(s) & =-m v^{2} \frac{1}{v^{2}}\left(g \boldsymbol{e}_{3}^{T}(\boldsymbol{\gamma})-\lambda \boldsymbol{N}(\boldsymbol{\gamma})\right) \\
& =m\left(-g \boldsymbol{e}_{3}+g\left(\boldsymbol{e}_{3} \cdot \boldsymbol{\gamma}\right) \boldsymbol{\gamma}-\frac{g}{\mu}\left(\boldsymbol{e}_{3} \cdot \boldsymbol{\gamma}\right) \boldsymbol{N}(\boldsymbol{\gamma}(t))\right) \\
& =-m g \boldsymbol{e}_{3}+m \lambda \boldsymbol{N}(\boldsymbol{\gamma}(s))-m \lambda \mu \boldsymbol{\gamma}(s) .
\end{aligned}
$$

From the equation above, we conclude that the normal force imparted by the ramp onto the block has magnitude $m \lambda$. Notice that the last equation above is Newton's second law.

It is clear that the form of the ramp depends on the desired constant speed of the block. For example, if the block is to travel upside down on some portion of the ramp and the speed $v$ is not much, then the curvature of that portion of the ramp must be big so that the block does not fall off the ramp. The following corollary explains how to change the ramp if we want to change the constant speed or if we want to change the gravity force: 
Corollary 4.2. Suppose that a ramp $\boldsymbol{R} \subset \mathbb{R}^{3}$ allows a block to move down with constant speed $v$ under a gravity $g$. Then, for any positive $\kappa$, the ramp $\kappa \boldsymbol{R}=$ $\{\kappa(x, y, z):(x, y, z) \in \boldsymbol{R}\}$ allows a block to move down

(a) with constant speed $\sqrt{\kappa} v$ and gravity $g$, or

(b) with constant speed $v$ and gravity $g / \kappa$.

Proof. This corollary is a consequence of the definition of the vector field $\boldsymbol{X}$ in (4-1). We can check that if $\boldsymbol{\gamma}(t)$ is an integral curve of the vector field $\boldsymbol{X}$ and $\boldsymbol{\alpha}(s)=\int_{0}^{s} \boldsymbol{\gamma}(u) d u$, then $\tilde{\boldsymbol{\gamma}}(\tau)=\boldsymbol{\gamma}(\tau / \kappa)$ is a solution of the vector field $(1 / \kappa) \boldsymbol{X}$, which can be interpreted as either the vector field coming from the tangent unit vector field $N(y)$ with velocity $\sqrt{\kappa} v$ and gravity $g$ or can be interpreted as the vector field coming from the tangent unit vector field $N(y)$ with velocity $v$ and gravity $g / \kappa$. The result follows by noticing that

$$
\tilde{\boldsymbol{\alpha}}(s)=\int_{0}^{s} \tilde{\boldsymbol{\gamma}}(u) d u=\int_{0}^{s} \boldsymbol{\gamma}\left(\frac{u}{\kappa}\right) d u=\kappa \int_{0}^{s / \kappa} \boldsymbol{\gamma}(v) d v=\kappa \boldsymbol{\alpha}\left(\frac{s}{\kappa}\right) .
$$

Remark 4.3. If a ramp $\boldsymbol{R}$ on earth has the property that an object will fall down with constant speed $v$, then, if we dilate this ramp by a factor of 6 , the same block will move down with the same constant speed $v$ on this dilated ramp when it is placed on the moon.

\section{Appendix: Graphs}

The graphs show snapshots of the motion on the longer part of the ramps, followed by snapshots of the motion on the shorter part. For a proper animation and explanatory video, see http://youtu.be/iBrvbb0efVk.

LONGER PART

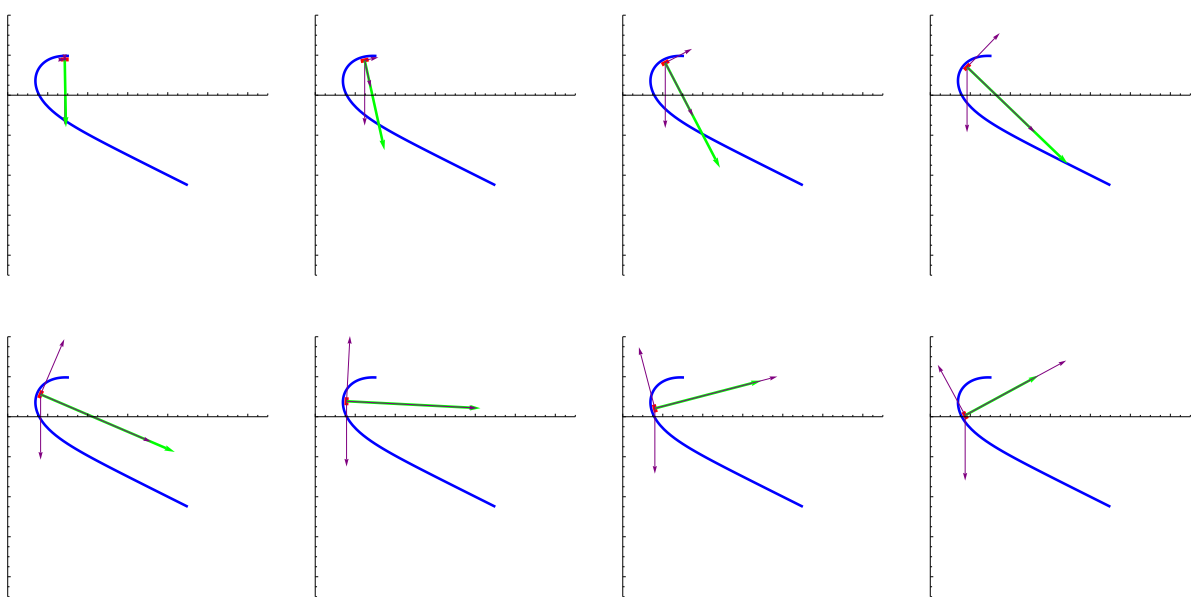


CONSTANT-SPEED RAMPS

17
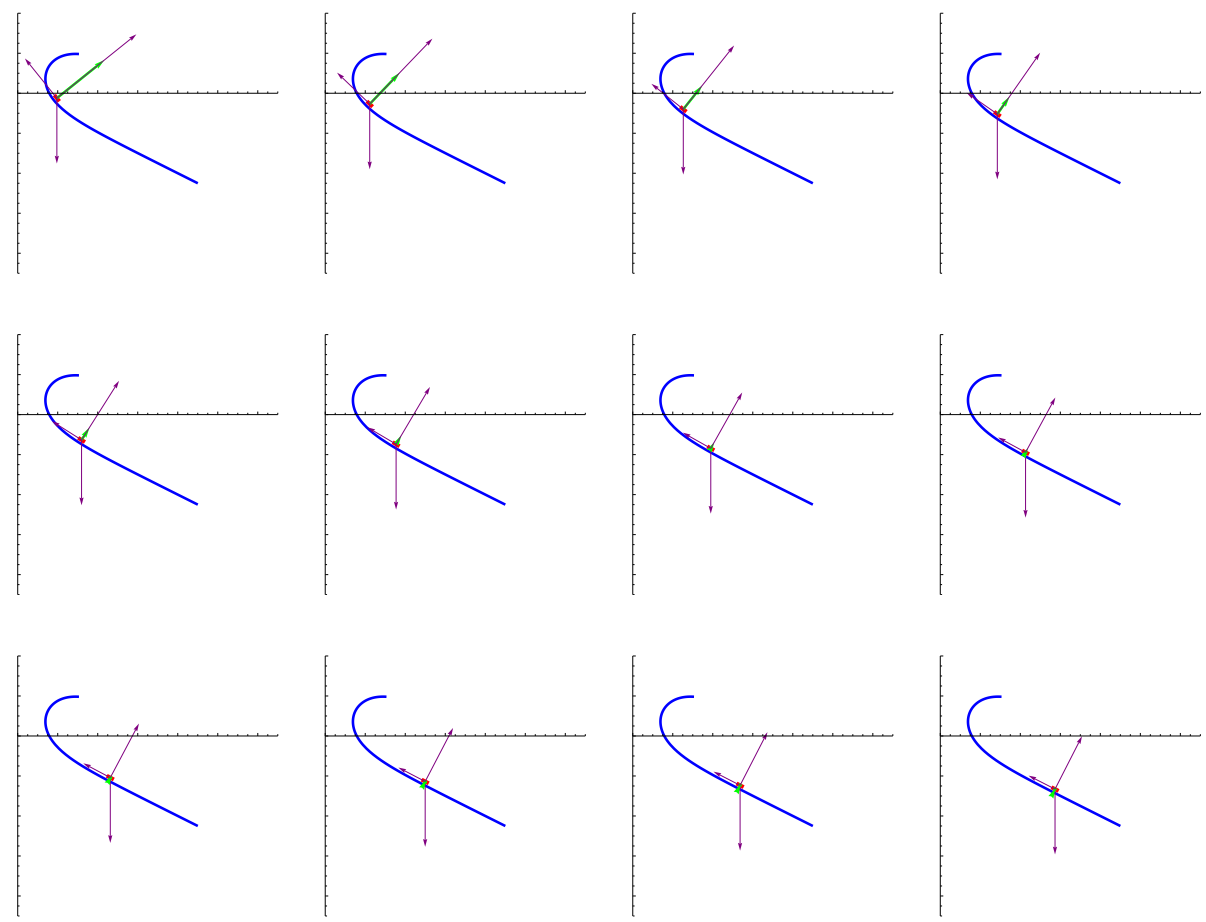

SHORTER PART
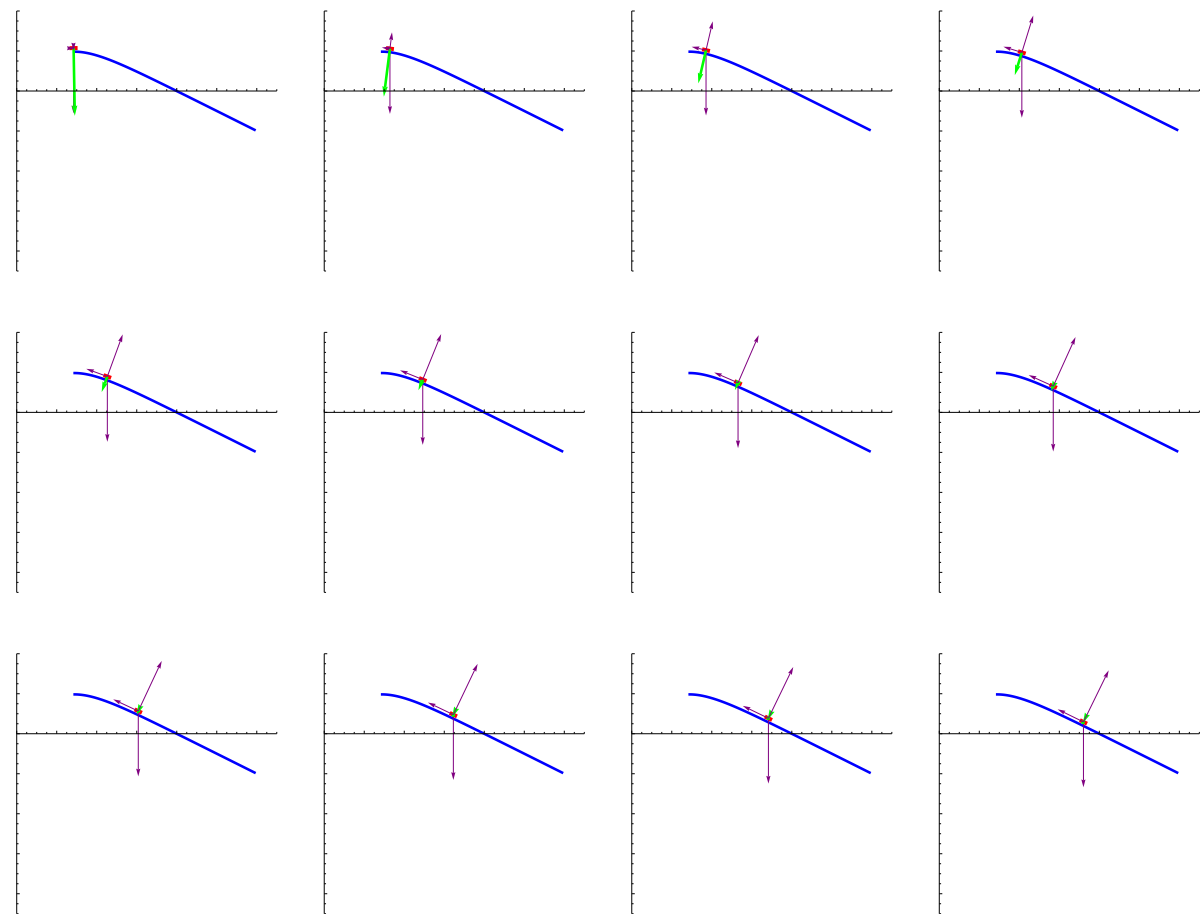


\section{Acknowledgements}

The author would like to express his gratitude to Frank Gould, Roger Vogeler, Nidal Al-Masoud and Clifford Anderson for their valuable comments on this work. $\mathrm{He}$ would also like to thank the referee for several comment that helped improve this paper.

\section{References}

[Beer et al. 2010] F. P. Beer, E. R. Johnston, D. F. Mazurek, and P. J. Cornwell, Vector mechanics for engineers: Statics and dynamics, 9th ed., McGraw-Hill, New York, 2010.

[do Carmo 1976] M. do Carmo, Differential geometry of curves and surfaces, Prentice-Hall, Englewood Cliffs, NJ, 1976. MR 52 \#15253 Zbl 0326.53001

[Feynman et al. 1963] R. P. Feynman, R. B. Leighton, and M. Sands, The Feynman lectures on physics, I: Mainly mechanics, radiation, and heat, Addison-Wesley, Reading, MA, 1963. MR 35 \#3942 Zbl 0131.38703

Received May 10, 2013. Revised October 5, 2013.

Oscar M. Perdomo

DEPARTMENT OF MATHEMATICS

Central Connecticut State University

NEW BRITAIN, CT 06050

UNITED STATES

perdomoosm@ccsu.edu 


\title{
PACIFIC JOURNAL OF MATHEMATICS
}

\author{
msp.org/pjm
}

Founded in 1951 by E. F. Beckenbach (1906-1982) and F. Wolf (1904-1989)

\section{EDITORS}

Don Blasius (Managing Editor)

Department of Mathematics

University of California

Los Angeles, CA 90095-1555

blasius@math.ucla.edu

\author{
Paul Balmer \\ Department of Mathematics \\ University of California \\ Los Angeles, CA 90095-1555 \\ balmer@math.ucla.edu \\ Robert Finn \\ Department of Mathematics \\ Stanford University \\ Stanford, CA 94305-2125 \\ finn@math.stanford.edu \\ Sorin Popa \\ Department of Mathematics \\ University of California \\ Los Angeles, CA 90095-1555 \\ popa@math.ucla.edu
}

\author{
Vyjayanthi Chari \\ Department of Mathematics \\ University of California \\ Riverside, CA 92521-0135 \\ chari@math.ucr.edu \\ Kefeng Liu \\ Department of Mathematics \\ University of California \\ Los Angeles, CA 90095-1555 \\ liu@math.ucla.edu \\ Jie Qing \\ Department of Mathematics \\ University of California \\ Santa Cruz, CA 95064 \\ qing@ cats.ucsc.edu
}

\section{PRODUCTION}

Silvio Levy, Scientific Editor, production@msp.org

\section{SUPPORTING INSTITUTIONS}

ACADEMIA SINICA, TAIPEI

CALIFORNIA INST. OF TECHNOLOGY

INST. DE MATEMÁTICA PURA E APLICADA

KEIO UNIVERSITY

MATH. SCIENCES RESEARCH INSTITUTE

NEW MEXICO STATE UNIV.

OREGON STATE UNIV.

\author{
STANFORD UNIVERSITY \\ UNIV. OF BRITISH COLUMBIA \\ UNIV. OF CALIFORNIA, BERKELEY \\ UNIV. OF CALIFORNIA, DAVIS \\ UNIV. OF CALIFORNIA, LOS ANGELES \\ UNIV. OF CALIFORNIA, RIVERSIDE \\ UNIV. OF CALIFORNIA, SAN DIEGO \\ UNIV. OF CALIF., SANTA BARBARA
}

\author{
Daryl Cooper \\ Department of Mathematics \\ University of California \\ Santa Barbara, CA 93106-3080 \\ cooper@math.ucsb.edu \\ Jiang-Hua Lu \\ Department of Mathematics \\ The University of Hong Kong \\ Pokfulam Rd., Hong Kong \\ jhlu@maths.hku.hk \\ Paul Yang \\ Department of Mathematics \\ Princeton University \\ Princeton NJ 08544-1000 \\ yang@math.princeton.edu
}

These supporting institutions contribute to the cost of publication of this Journal, but they are not owners or publishers and have no responsibility for its contents or policies.

See inside back cover or msp.org/pjm for submission instructions.

The subscription price for 2015 is US \$420/year for the electronic version, and \$570/year for print and electronic.

Subscriptions, requests for back issues and changes of subscribers address should be sent to Pacific Journal of Mathematics, P.O. Box 4163, Berkeley, CA 94704-0163, U.S.A. The Pacific Journal of Mathematics is indexed by Mathematical Reviews, Zentralblatt MATH, PASCAL CNRS Index, Referativnyi Zhurnal, Current Mathematical Publications and Web of Knowledge (Science Citation Index).

The Pacific Journal of Mathematics (ISSN 0030-8730) at the University of California, c/o Department of Mathematics, 798 Evans Hall \#3840, Berkeley, CA 94720-3840, is published twelve times a year. Periodical rate postage paid at Berkeley, CA 94704, and additional mailing offices. POSTMASTER: send address changes to Pacific Journal of Mathematics, P.O. Box 4163, Berkeley, CA 94704-0163.

PJM peer review and production are managed by EditFLOW ${ }^{\circledR}$ from Mathematical Sciences Publishers.

\section{PUBLISHED BY}

\section{mathematical sciences publishers \\ nonprofit scientific publishing}

http://msp.org/

(C) 2015 Mathematical Sciences Publishers 


\section{PACIFIC JOURNAL OF MATHEMATICS}

Volume $275 \quad$ No. $1 \quad$ May 2015

Constant-speed ramps

OSCAR M. PERDOMO

Surfaces in $\mathbb{R}_{+}^{3}$ with the same Gaussian curvature induced by the

Euclidean and hyperbolic metrics

Nilton BARRoso and PEDRO RoITMAN

Cohomology of local systems on the moduli of principally polarized abelian surfaces

DAN PETERSEN

On certain dual $q$-integral equations

Ola A. Ashour, Mourad E. H. Ismail and Zeinab S.

MANSOUR

On a conjecture of Erdôs and certain Dirichlet series

TAPAS CHATTERJEE and M. RAM MURTY

Normal forms for CR singular codimension-two Levi-flat submanifolds

XiAnGHONG GONG and JiŘí LEBL

Measurements of Riemannian two-disks and two-spheres

FLORENT BALACHEFF

Harmonic maps from $\mathbb{C}^{n}$ to Kähler manifolds

JIANMING WAN

Eigenvarieties and invariant norms

Claus M. Sorensen

The Heegaard distances cover all nonnegative integers 